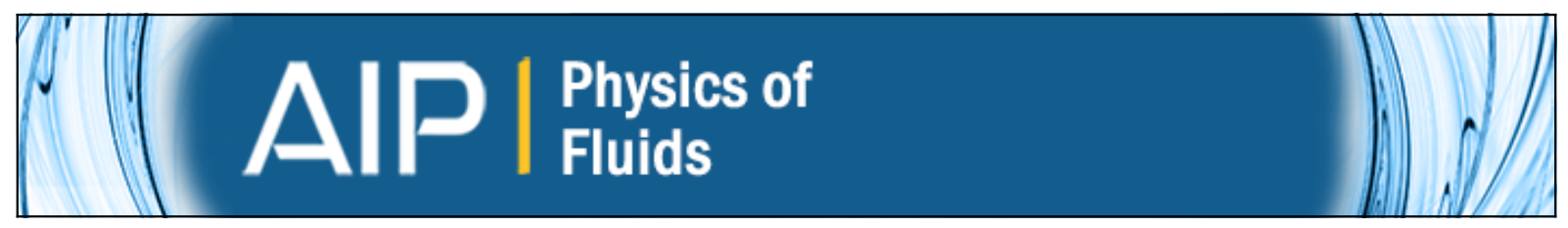

Inlet conditions for wave packet models in turbulent jets based on eigenmode decomposition of large eddy simulation data

Daniel Rodríguez, Aniruddha Sinha, Guillaume A. Brès, and Tim Colonius

Citation: Physics of Fluids (1994-present) 25, 105107 (2013); doi: 10.1063/1.4824479

View online: http://dx.doi.org/10.1063/1.4824479

View Table of Contents: http://scitation.aip.org/content/aip/journal/pof2/25/10?ver=pdfcov

Published by the AIP Publishing

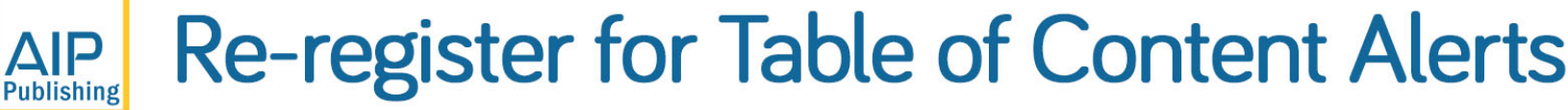




\title{
Inlet conditions for wave packet models in turbulent jets based on eigenmode decomposition of large eddy simulation data
}

\author{
Daniel Rodríguez,, ${ }^{1,2, a)}$ Aniruddha Sinha, ${ }^{1}$ Guillaume A. Brès, ${ }^{3}$ \\ and Tim Colonius ${ }^{1}$ \\ ${ }^{1}$ Division of Engineering and Applied Science, California Institute of Technology, \\ Pasadena, California 91125, USA \\ ${ }^{2}$ E. T. S. I. Aeronáuticos, Universidad Politécnica de Madrid, Pza. Cardenal Cisneros 3, \\ E-28040 Madrid, Spain \\ ${ }^{3}$ Cascade Technologies Inc., Palo Alto, California 94303, USA
}

(Received 28 March 2013; accepted 24 September 2013; published online 15 October 2013)

\begin{abstract}
This paper makes contributions towards reduced-order models of wave packets in supersonic, turbulent jets. Wave packets are large-scale turbulent structures that are correlated and advected over distances that are large compared to the integral scales of turbulence, i.e., many jet diameters at the lowest frequencies. They are thought to be responsible for the peak noise radiated at shallow angles to the jet axis. Linear wave packet models based on the Parabolized Stability Equations (PSE) have been shown in the past to be in excellent agreement with statistical structures educed from experimental pressure and velocity data in subsonic jets. Here, we extend these models to supersonic jets and validate them using a Large Eddy Simulation (LES) database for an isothermal and a moderately heated Mach 1.5 turbulent jets. For supersonic jets, inlet conditions for PSE models are ambiguous, as a parallel flow stability analysis shows several unstable modes at the inlet cross section. We develop a bi-orthogonal decomposition and project the LES data onto the relevant families of instability waves. These serve as inlet conditions, including the amplitude and shape functions, for PSE solutions which are then favorably compared to the near-field pressure fields educed from LES. @ 2013 AIP Publishing LLC. [http://dx.doi.org/10.1063/1.4824479]
\end{abstract}

\section{INTRODUCTION}

Reducing the noise emitted by turbulent jets is a technological problem that has received continuous effort since the appearance of commercial jet airliners in the early 1950s. Numerical simulations are capable of recovering both the properties of the highly turbulent mixing flow and its radiated sound with remarkable accuracy. However, a complete theory describing the generation of turbulent mixing noise that would permit the elaboration of simplified models for noise prediction and control is still lacking. Over the last decades, a theory based on the existence and dynamics of wave packets as the prominent noise sources has been elaborated, and extensive comparison with data from simulations and experiments demonstrated its utility. Jordan and Colonius ${ }^{1}$ review the wave packet's theoretical framework and supporting experimental evidence.

Wave packets are intermittent advected disturbances within the turbulent mixing flow that are correlated over significant distances along the jet axial direction, thus serving as efficient noise sources. Evidence has been amassed that the peak noise emitted by jets, taking place at relatively low frequencies and being highly directional, is related to these coherent structures. The noise radiated to the acoustic far-field is mostly contained in the first few $(m=0,1$, and 2$)$ azimuthal wavenumbers, ${ }^{2}$

\footnotetext{
a) Author to whom correspondence should be addressed. Electronic mail: dani@ torroja.dmt.upm.es. Present address: Escola de Engenharia de São Carlos, Universidade de São Paulo, Brazil.
} 
whereas the turbulent kinetic energy fluctuations are spread over a significant range of wavenumbers and peak at higher values of $m$. However, relatively high correlations between the azimuthally decomposed velocity field measured using time-resolved Particle Image Velocimetry and far-field pressure measurements suggest that the peak noise radiation is generated by the lower azimuthal wavenumbers, regardless of their relatively small energy. ${ }^{3}$ Consequently, if an adequate prediction of the wave packets corresponding to the first few azimuthal modes at lower frequencies can be obtained, these would provide a useful reduced-order model to guide noise reduction strategies.

Theories modeling wave packets as instability waves of the mean turbulent flow were discussed by Crighton and Gaster. ${ }^{4}$ Most of these attempts consider statistical descriptions of the wave packets in the frequency domain, permitting the derivation of a set of equations that describe the evolution of each individual frequency and azimuthal mode, while inter-modal nonlinear interactions appear as Reynolds stresses. However, as was pointed out by Crighton and Gaster ${ }^{4}$ and Mankbadi and Liu, ${ }^{5}$ when interest is focused on the low frequencies and low azimuthal wavenumbers, the Reynolds stresses acting on them may be negligible due to their small energy content. A linear model for the individual modes is then justified, assuming that the overall effect of the nonlinearity, fundamental to the process of turbulent mixing, is already accounted for in the establishment of the turbulent mean flow. A further improvement in the linear model can be achieved with the introduction of $a d$ hoc eddy viscosity models. ${ }^{5-7}$

Following diverse assumptions on the characteristics of the mean flow and wave packets, different approaches to the computation of instability waves have been employed in the past, including locally parallel stability analysis based on the Orr-Sommerfeld equation, ${ }^{8}$ multiple-scales analysis,,${ }^{4,9}$ parabolized stability equations (PSE),${ }^{10-13}$ global eigenmode analysis, ${ }^{14}$ and boundaryvalue problems ${ }^{15}$ Independent of the particular approach followed for the wave packet modeling, the governing equations are deterministic and the solution depends on prescribed inlet conditions. Furthermore, when natural (unforced) jets are considered, the inlet conditions are known only statistically and the complete wave packet description depends on the disturbance spectrum at the inlet of the computational domain. Data from experiments and high-fidelity numerical simulations can be used in order to fill the gap in the modeling process, determining adequate inlet conditions for the wave packet models and consequently calibrating their amplitudes in order to serve as predicting tools. In line with this, numerical simulations present two main advantages over experiments: all the fluid variables are computed, as opposed to experiments in which individual quantities (velocity, pressure, etc.) are measured, and the data are made available simultaneously at a large number of spatial locations with adequate temporal resolution.

The usual practice for the determination of inlet conditions for numerical simulations ${ }^{16,17}$ and PSE relies on forcing by eigenfunctions of the classical linear stability theory (LST) of locally parallel flows. LST studies the growth or decay of perturbation waves about a base flow assumed to be homogeneous along the streamwise direction, permitting to recast the equations governing the perturbations at any given cross section into a local eigenvalue problem (EVP). Typically, only the eigensolution for each frequency and azimuthal number exhibiting the largest spatial amplification, i.e., the one corresponding to Kelvin-Helmholtz (K-H) instability, is considered, ignoring the contribution of the other families of eigenmodes present in the spectrum. This is partially justified by the fact that, regardless of the particularities of the inlet disturbance spectrum, the most amplified eigenmode is expected to dominate the disturbance field past a short distance downstream from the nozzle lip. However, the amplitude of the LST eigenfunction still needs to be determined. Tam and $\mathrm{Chen}^{18}$ proposed a method for the determination of the stochastic wave packet amplitudes, by assuming that the $\mathrm{K}-\mathrm{H}$ instability wave was representative of all the fluctuation energy content at each frequency and matching it to the turbulent kinetic energy at the nozzle lip.

Tam and $\mathrm{Hu}^{19}$ computed families of spatially amplified eigensolutions under the parallel flow approximation for high-speed subsonic and supersonic jet flows showing that unstable solutions different from the dominant K-H instability are present in supersonic jets. These explain the crosshatched radiation patterns observed experimentally by Oertel $^{20}$ and may be related to the generation of screech tones in imperfectly expanded jets. In addition, LST shows that several families of neutral and damped eigensolution exist, besides the amplified wave solutions. 
In order to account for the complete spectrum of inlet perturbations in their computations of linear wave packets and Mach wave radiation, Mohseni et al. ${ }^{15}$ imposed the perturbation profile computed from a previous direct numerical simulation as an inlet condition.

The decomposition of a total fluctuating flow field delivered by numerical simulation into the separate contributions of the different eigenmode families may provide further insight into the wave packet physics. In particular, it can be used to determine the leading contributions that are relevant to the wave packets and those corresponding to physical mechanisms that, while possibly dominating the fluctuation field in some spatial regions, are meaningless to the wave packet evolution. This eigenmode decomposition can be achieved by making use of the bi-orthogonality between the eigenfunctions of the direct and adjoint LST problems. ${ }^{21-23}$

The approach of modeling wave packets as linear instability waves has been widely employed in the study of forced supersonic jets, $, 10,12,24-26$ for which the measured near-field fluctuations were found to be in good agreement with the predictions of linear stability theory. ${ }^{9}$ In the case of subsonic natural jets, this approach has only recently begun to deliver satisfactory quantitative predictions, both in the near pressure and velocity fields. ${ }^{3,13,27}$ One of the difficulties associated with the empirical observation of the coherent structures in natural jets is the lack of a phase reference that is trivially determined in forced jets. Advanced measurement technologies such as phased microphone arrays and time-resolved particle image velocimetry, along with cross-correlation techniques like Proper Orthogonal Decomposition (POD) were instrumental in extracting the coherent part of the turbulent motion, thus enabling the comparison and demonstrating the utility of the approach. Comparisons considering simulations or experiments of unforced supersonic jets with linear wave packet models are still scarce in the literature: Ray et al. ${ }^{7}$ compared the phase velocity of PSE solutions with pressure measurements for a $M_{j}=1.41$, underexpanded isothermal jet by using two-point correlations, but comparisons of the wave packet evolution along the axial direction have not been reported for unforced jets with supersonic exhaust speed.

Details of the jet configurations, LES computations and post-processing are given in Sec. II. Section III describes the theoretical background for the wave packet computation using PSE and the derivation of the local EVP and the eigenmode decomposition technique. The properties of the eigenspectrum and the different families of eigenfunctions predicted by linear stability theory in the vicinity of the nozzle lip, where inlet conditions are imposed, are studied in Sec. IV. The projection of fluctuation profiles extracted from the LES database on the different families of LST eigenmodes is performed in Sec. V, where the relevance of the different contributions to the PSE inlet conditions is also discussed. In Sec. VI, the derived inlet conditions are used in the computation of wave packets using PSE, and the results are compared quantitatively with the averaged and POD-filtered near-field pressure from the simulation, showing favorable agreement. The conclusions drawn are summarized in Sec. VII.

\section{LARGE EDDY SIMULATION DATABASE AND PROCESSING}

Large eddy simulation data corresponding to two ideally expanded supersonic jets emanating from a convergent-divergent nozzle are used in the present work. The jet Mach number defined by the jet exit velocity and speed of sound is $M_{j}=U_{j} / c_{j}=1.5$ for both conditions. The first case corresponds to an isothermal jet with static temperature ratio $T_{j} / T_{\infty}=1.0$, where subscript $j$ refers to the jet exit conditions and $\infty$ to the ambient conditions. The Reynolds number defined by the jet exit velocity, density $\rho_{j}$, and nozzle diameter $D$ is $R e=300000$ for the isothermal jet. The second configuration, referred to as hot jet, is a moderately heated $\left(T_{j} / T_{\infty}=1.74\right)$ jet at $R e=155000$. In both cases, the fluid surrounding the jet has a small co-flow component $\bar{u}_{c o}=u_{c o} / c_{\infty}=0.1$ which is chosen to match the corresponding experimental conditions in the United Technologies Research Center (UTRC) anechoic facility. ${ }^{28}$ The LES computations were performed using Cascade Technologies' flow solver "Charles" on an axisymmetric unstructured mesh containing approximately $42 \times 10^{6}$ control volumes, with mostly hexahedral elements. "Charles" uses a finite volume method on an unstructured grid with localized adaptive refinement. Vreman's subgrid model is used in order to account for the unresolved scales. No perturbations are prescribed inside the nozzle, resulting in a thin transitional shear-layer at the nozzle lip that becomes fully turbulent over the first diameter. Extensive 
comparisons of the simulation results against measurements performed at UTRC, considering mean flow quantities and near- and far-field pressure spectra, showed excellent agreement. More details on the LES simulation and its validation can be found in Brès et al. ${ }^{29}$

In what follows, physical quantities are made dimensionless with the jet diameter $D$, the ambient speed of sound $c_{\infty}$, and the ambient density $\rho_{\infty}$. The dimensionless frequency used to show results will be the Strouhal number defined as $S t=f D / U_{j}$.

Time-averaged mean flow variables were computed using the total simulation time (after initial transients) of $t_{\text {tot }} \approx 215 \mathrm{D} / c_{\infty}$ for the isothermal jet and $112 \mathrm{D} / c_{\infty}$ for the hot jet, considered to be a long time sample of high-fidelity LES, thus ensuring the statistical convergence of the stationary quantities and also a reasonable convergence of the low-frequency noise spectra. The fluctuation profiles used in the eigenmode projection are obtained from the discrete Fourier transform (DFT) of the LES data. The time segments used in the DFTs correspond to the frequency bin $\Delta S t=0.025$ and are overlapped $75 \%$ of their length, resulting in 29 and 19 segments for the isothermal and hot jets, respectively.

\section{THEORETICAL BACKGROUND}

The turbulent flow field is decomposed into a time-averaged (or mean) flow and temporal fluctuations, $\mathbf{q}(\mathbf{x}, t)=\overline{\mathbf{q}}(\mathbf{x})+\mathbf{q}^{\prime}(\mathbf{x}, t)$. A cylindrical coordinate system is used where $\mathbf{x}=(x, r, \theta)$ are the axial, radial, and azimuthal coordinates, respectively. The vector of fluid variables is denoted by $\mathbf{q}=\left[u_{x}, u_{r}, u_{\theta}, p, \zeta\right]$, where $u_{x}, u_{r}$, and $u_{\theta}$ are the axial, radial, and azimuthal velocity components, $p$ is pressure, and $\zeta=1 / \rho$ is the specific volume.

Assuming statistical stationarity and azimuthal homogeneity, the mean flow is only function of the axial and radial coordinates $(\overline{\mathbf{q}}=\overline{\mathbf{q}}(x, r))$ and Fourier modes are introduced for frequency $\omega=2 \pi M_{j} S t$ and wavenumber $m$ as

$$
\mathbf{q}^{\prime}(\mathbf{x}, t)=\sum_{\omega} \sum_{m} \breve{\mathbf{q}}_{\omega, m}(x, r) \mathrm{e}^{\mathrm{i} m \theta} \mathrm{e}^{-\mathrm{i} \omega t} .
$$

\section{A. Parabolized stability equations}

Owing to the slow variation of the mean flow along the axial direction (compared to the radial variation), PSEs have been used in the past ${ }^{3,7,12,13}$ as a model for the spatial evolution of the largescale wave packets. Based on the multiple-scales approach, ${ }^{4}$ PSE accounts for the slow spread of the jet mean flow and delivers results comparable to spatial direct numerical simulations for convectively unstable flows. ${ }^{30}$ The fluctuations $\breve{\mathbf{q}}_{\omega, m}(x, r)$ are decomposed into a shape function $\tilde{\mathbf{q}}_{\omega, m}$ which is slowly varying along the axial direction, evolving on the same scale as the mean flow, and a rapidly varying wave-like part:

$$
\begin{aligned}
\breve{\mathbf{q}}_{\omega, m} & =A_{\omega, m}(x) \tilde{\mathbf{q}}_{\omega, m}(x, r) \\
& =A_{\omega, m}\left(x_{0}\right) \exp \left(\mathrm{i} \int_{x_{0}}^{x} \alpha_{\omega, m}(\xi) d \xi\right) \tilde{\mathbf{q}}_{\omega, m}(x, r) .
\end{aligned}
$$

The axial wavenumber $\alpha_{\omega, m}=\alpha_{r}+\mathrm{i} \alpha_{i}$ is a complex quantity for which a slow variation has also been assumed. The axial coordinate $x_{0}$ corresponds to the location where the PSE integration is initialized, typically a cross-section close to the nozzle lip. In the present computations $x_{0}=0.5 \mathrm{D}$, which is close enough to the nozzle to obtain consistent results. This issue is discussed further in Sec. VI. Introducing this decomposition into the compressible Navier-Stokes, continuity and energy equations and subtracting the terms corresponding to the mean flow we arrive at the system of equations

$$
\left(\mathbf{A}+\frac{d \alpha}{d x} \mathbf{B}+\mathbf{C} \frac{\partial}{\partial x}+\mathbf{D} \frac{\partial}{\partial r}+\mathbf{E} \frac{\partial^{2}}{\partial r^{2}}+\mathbf{F} \frac{\partial^{2}}{\partial x \partial r}\right) \tilde{\mathbf{q}}_{\omega, m}(x, r)=R_{\omega, m} .
$$

The linear operators $\mathbf{A}$ to $\mathbf{E}$ depend on the mean flow quantities, Reynolds number, Mach number, frequency $\omega$, azimuthal wavenumber $m$, and axial wavenumber $\alpha$. Details on the derivation of the 
system of equations (3) can be found elsewhere. ${ }^{13,31}$ The system of equations (3) is a linear spatial operator for each mode $(\omega, m)$ with the forcing term $R_{\omega, m}$ accounting for mode-dependent Reynolds stresses. For unforced turbulent jets, the small relative amplitude of the individual modes suggests that nonlinear interactions between the lower modes can be neglected, as most nonlinear effects are implicit in the mean flow, so that $R_{\omega, m} \approx 0 .{ }^{4,5}$

After spatial discretization of the radial direction, the system (3) takes the form

$$
\mathbf{L} \frac{\partial \tilde{\mathbf{q}}_{\omega, m}}{\partial x}=\mathbf{R} \tilde{\mathbf{q}}_{\omega, m},
$$

where $\mathbf{L}=\mathbf{C}+\mathbf{F} \mathcal{D}_{r}$ and $\mathbf{R}=-\left(\mathbf{A}+d \alpha / d x \mathbf{B}+\mathbf{D} \mathcal{D}_{r}+\mathbf{E} \mathcal{D}_{r r}\right)$ have been introduced. The matrix operators $\mathcal{D}_{r}$ and $\mathcal{D}_{r r}$ stand for the discrete versions of the first and second order spatial differentiation along the radial direction, respectively, and also account for the boundary conditions. The numerical differentiation scheme and the boundary conditions are discussed in Appendix A. The subscripts $(\omega$, $m$ ) have dropped for simplicity of notation.

The decomposition of (2) is ambiguous in that the spatial growth can be absorbed into the shape function $\tilde{\mathbf{q}}_{\omega, m}$ or the complex amplitude $A_{\omega, m}$. Following Herbert, ${ }^{30}$ the normalization condition

$$
\int_{0}^{\infty} \tilde{\mathbf{u}}_{\omega, m}^{*} \frac{\partial \tilde{\mathbf{u}}_{\omega, m}}{\partial x} r d r=0,
$$

where $\tilde{\mathbf{u}}_{\omega, m}$ refers to the vector of three velocity components and $*$ denotes complex conjugation, is imposed individually to every $(\omega, m)$ mode, removing the exponential dependence from $\tilde{\mathbf{q}}_{\omega, m}$.

Expression (4) is an initial value problem (with the axial distance, $x$, as a time-like variable) to be integrated along the axial direction. It requires inlet conditions for the shape function $\tilde{\mathbf{q}}_{\omega, m}$ and amplitude $A_{\omega, m}$ at some location $x_{0}$. Information provided by locally parallel linear stability theory (discussed below) is typically employed in the determination of the inlet conditions.

\section{B. Local stability eigenvalue problem}

A local stability eigenvalue problem (EVP) is derived here from the PSE approximation, by assuming $d \alpha_{\omega, m} / d x \approx 0$ and $\partial \breve{\mathbf{q}}_{\omega, m} / \partial x \approx \mathrm{i} \alpha_{\omega, m} \breve{\mathbf{q}}_{\omega, m}$. These assumptions are the usual ones in the derivation of local stability problems of the Orr-Sommerfeld kind, but two differences exist in the present approach. First, the axial derivatives of the mean flow that are retained in PSE are also retained here. As shown by Gudmundsson and Colonius, ${ }^{13}$ these terms have a very minor effect on the results. Second, the second derivatives of the perturbations in the axial direction appearing in the viscous term are neglected here as is done in PSE. These terms yield $\alpha^{2}$ terms in the usual spatial stability problem, requiring a special treatment to recast the equations as a linear EVP that doubles the size of the matrix EVP. Neglecting these terms alters the results from LST by eliminating two branches of upstream propagating vortical and entropic waves in the eigenspectrum, as shown by $\mathrm{Li}$ and Malik, ${ }^{32}$ leaving the other families practically unaltered. The local EVP resulting from these approximations does not correspond exactly to the parallel-flow stability approach as some of the first order non parallel terms are kept. However, this formulation is chosen to arrive at an EVP which is consistent with the PSE operators, also permitting the use of the same characteristic boundary conditions at the outer flow.

From the approximations above, the following matrix EVP results:

$$
i \alpha \mathbf{L} \hat{\mathbf{q}}=\mathbf{R} \hat{\mathbf{q}},
$$

where the subscripts $(\omega, m)$ have been dropped for simplicity. Operators $\mathbf{L}$ and $\mathbf{R}$ are those in (4), but particularized for $\alpha=0$. This property is demonstrated in Appendix B. The eigenvalue problem (6) describes the spatial growth or decay of disturbance waves with a fixed real frequency $\omega$ and azimuthal wavenumber $m$. In addition to the Kelvin-Helmholtz eigenmode, there is a full eigenspectrum of solutions that is usually ignored when computing initial conditions for the PSE integration, but is necessary in order to expand an arbitrary perturbation. The eigenspectrum obtained as solution of the differential problem (6) has discrete and continuous branches, but the spatial discretization of the problem leads to a discretization of the continuous branches, and consequently only numerable and 
discrete eigenmodes are obtained. To avoid confusion with the notation used so far, the eigenfunction and eigenvalue corresponding to eigenmode $n$ will be referred to as $\hat{\mathbf{q}}_{n}$ and $\hat{\alpha}_{n}$ in what follows. The properties of the eigenspectrum and eigenfunctions will be discussed in Sec. IV.

\section{Bi-orthogonal decomposition and adjoint LST problem}

As shown for flat plate boundary layer flows, ${ }^{21,23,33,34}$ the eigenspectrum from LST is a complete basis and an arbitrary perturbation profile can be decomposed as a linear superposition of the eigenfunctions $\breve{\mathbf{q}}_{0}(r)=\sum_{n} a_{n} \hat{\mathbf{q}}_{n}(r)$. Due to the non-normality of the linearized Navier-Stokes equations, the eigenfunctions do not form an orthogonal system, and the solutions of the adjoint problem are required in order to obtain a bi-orthogonality relation. The discrete adjoint problem, rather than the analytical adjoint used in the references, is employed here in order to simplify the computations. Using the usual scalar product between complex vectors, the adjoint problem is

$$
\mathrm{i} \alpha^{+} \mathbf{L}^{H} \hat{\mathbf{q}}^{+}=\mathbf{R}^{H} \hat{\mathbf{q}}^{+},
$$

where the superscript $H$ denotes Hermitian transposed and + denotes adjoint variables. The adjoint eigenfunctions will be referred to as $\hat{\mathbf{q}}_{n}^{+}$. The adjoint eigenvalues $\alpha_{n}^{+}$are related to those of the direct problem (6) by $\mathrm{i} \alpha_{n}=\left(\mathrm{i} \alpha_{n}^{+}\right)^{*}$. By construction, the bi-orthogonality relation

$$
\left(\alpha_{n}-\alpha_{l}\right)\left(\hat{\mathbf{q}}_{n}^{+}\right)^{H} \mathbf{L} \hat{\mathbf{q}}_{l}=0
$$

can be used to obtain the weighting coefficients $a_{n}$ in the projection of an arbitrary perturbation profile $\breve{\mathbf{q}}_{0}(r)$ onto the primal eigenfunctions:

$$
a_{n}=\left(\left(\hat{\mathbf{q}}_{n}^{+}\right)^{H} \mathbf{L} \breve{\mathbf{q}}_{0}\right) /\left(\left(\hat{\mathbf{q}}_{n}^{+}\right)^{H} \mathbf{L} \hat{\mathbf{q}}_{n}\right) .
$$

The adjoint eigenfunctions illustrate the spatial regions where the different eigenmodes are more receptive and serve as a filter of the relative contribution of the individual eigenmodes onto the arbitrary perturbation profile. ${ }^{22,35,36}$

It should be noted that discrete adjoint formulations like the one used here may be inconsistent with boundary conditions assuming continuous derivatives, leading to adjoint eigenfunctions with unphysical behavior near the boundaries. In those cases, the computed coefficients $a_{n}$ take meaningless values and the eigenmode expansion does not converge. In the present computations, the combination of characteristic boundary conditions with a fine resolution at the outer boundary, as discussed in Appendix A, resulted in good behavior of the adjoint eigenfunctions at $r \rightarrow \infty$. Convergence of the eigenmode expansion is shown in Figure 4.

\section{PROPERTIES OF THE EIGENSPECTRUM}

The inviscid LST eigenspectrum was studied for jet profiles in Gill, ${ }^{37}$ Tam and Burton ${ }^{38}$ and Tam and Hu. ${ }^{19}$ The properties of the viscous eigenspectrum were studied in the framework of temporal LST by Bertolotti and Colonius ${ }^{39}$ for a $M_{j}=0.9$ jet, and the different families of eigenmodes were identified and used as initial conditions for the PSE integration. While the physics associated with the linear waves is the same in the spatial and temporal frameworks, the structure of the spatial eigenspectrum is different from Bertolotti and Colonius ${ }^{39}$ and is discussed here. Only the tophat velocity profiles representative of the nozzle lip region is considered here. The eigenspectrum properties are qualitatively identical for the supersonic cold and hot jets studied here.

The solution of the locally parallel linear stability EVP (6) delivers a complete eigenspectrum of waves. Each eigenmode family is related to a spatial region in the jet mean flow profile. The outer flow, i.e., the uniform unbounded stream surrounding the jet, supports a continuous spectrum of pressure (acoustic), vorticity, and entropy waves. The description of these families was done by Balakumar and Malik ${ }^{40}$ when considering the free-stream of compressible boundary-layers. The numerical treatment followed here leads to a discretization of the continuous branches; the particular set of eigenvalues recovered depends on the spatial discretization. Similarly, the potential core can be regarded as a bounded uniform flow, supporting infinite discrete equivalents of the continuous 

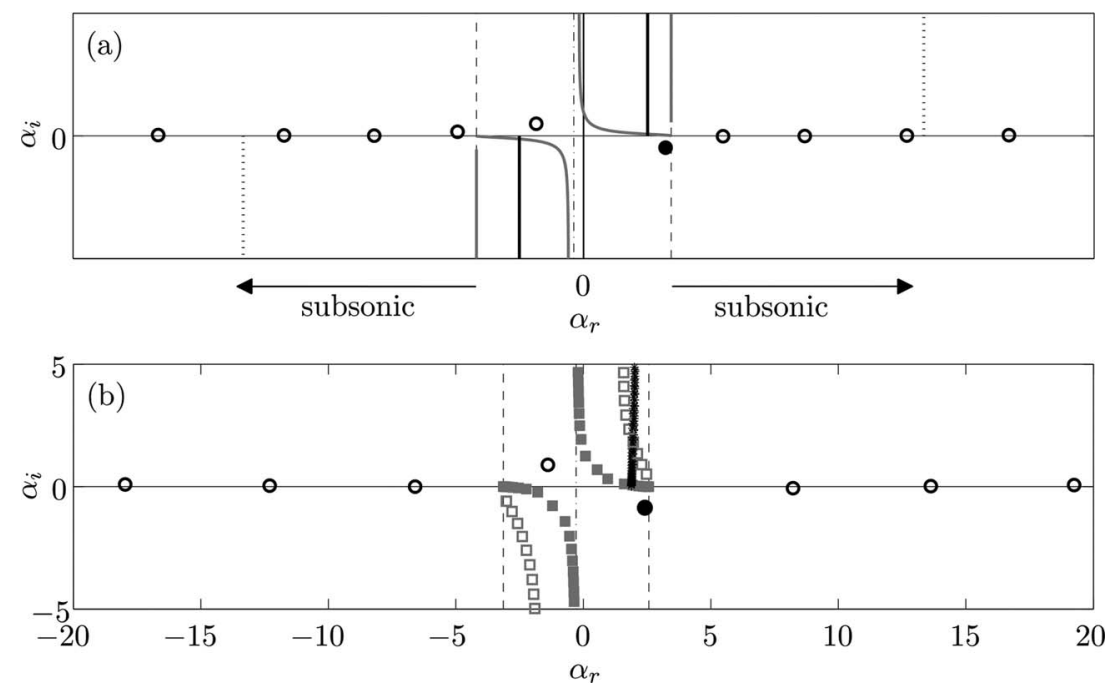

FIG. 1. Spatial LST eigenspectrum for a supersonic jet. (a) Sketch of the different branches of solution. (b) Eigenspectra for the isothermal $M_{j}=1.5$ jet at $x=0.5 D$, for $S t=0.3$ and $m=0$. Grey lines and symbols correspond to acoustic waves; vertical solid black lines and asterisks correspond to vorticity and entropy waves in the potential core; vertical dotted black lines correspond to vorticity and entropy waves in the outer flow; open black circles correspond to core pressure waves; and the full circle corresponds to the Kelvin-Helmholtz eigenmode.

spectra. Finally, the annular shear-layer gives rise to a discrete eigenmode corresponding to the K-H mechanism.

Figure 1 shows the structure of the eigenspectrum. The upper plot is a sketch of the theoretical continuous, discrete infinite, and K-H eigenmodes. The lower plot shows one representative eigenspectrum obtained using the mean profile of the isothermal jet at the axial location $x=0.5 \mathrm{D}$ for $S t$ $=0.3$ and $m=0$. The eigenspectrum is qualitatively identical for helical $(m \neq 0)$ modes.

\section{A. Instability waves}

Considering inviscid spatial instability theory, Tam and $\mathrm{Hu}^{19}$ determined three possible kinds of instability waves. The first kind corresponds to the K-H instability and exists for both subsonic and supersonic jets. The second and third kinds of instability waves can only exist for supersonic jets, and correspond to resonances (or reflections ${ }^{37}$ ) of the pressure waves within the potential core, similar to a Mach wave system confined by the annular mixing layer. Tam and $\mathrm{Hu}^{19}$ considered these pressure waves as two different kinds according to their subsonic or supersonic phase velocity with respect to the ambient, since their physical properties and experimental realizability differ notably. ${ }^{20}$

The same families of instability waves from Tam and $\mathrm{Hu}^{19}$ are found here, namely, the K-H eigenmode and a set of resonant pressure waves within the potential core. The phase velocity of the downstream propagating members of this set is subsonic with respect to the ambient speed, as was also predicted by Tam and $\mathrm{Hu}^{19}$ due to the relatively small (but supersonic) jet exhaust velocity. These modes, K-H and core pressure waves, are denoted by the filled and open circles in Figure 1. The axial velocity and pressure eigenfunctions corresponding to these modes, scaled with the axial velocity at the lipline, are shown in Figure 2 and are in perfect agreement with those in Tam and Hu. ${ }^{19}$ Inspection of their eigenfunctions suggests that they can be considered as a single branch, and their elements enumerated according to the number of peaks, $k$, present inside the potential core. The first one corresponds to K-H instability; it is characterized by a marked peak at the lipline and has no peaks inside the potential core $(k=0)$. Eigenmodes $k=1, k=2, \ldots$ are core pressure modes propagating downstream. In a similar fashion, the part of this branch with negative phase velocity (with $\alpha_{r}<0$ ) is denoted by $k=-1, k=-2$ and so on. While the $k=-1,-2, \ldots$ eigenmodes 

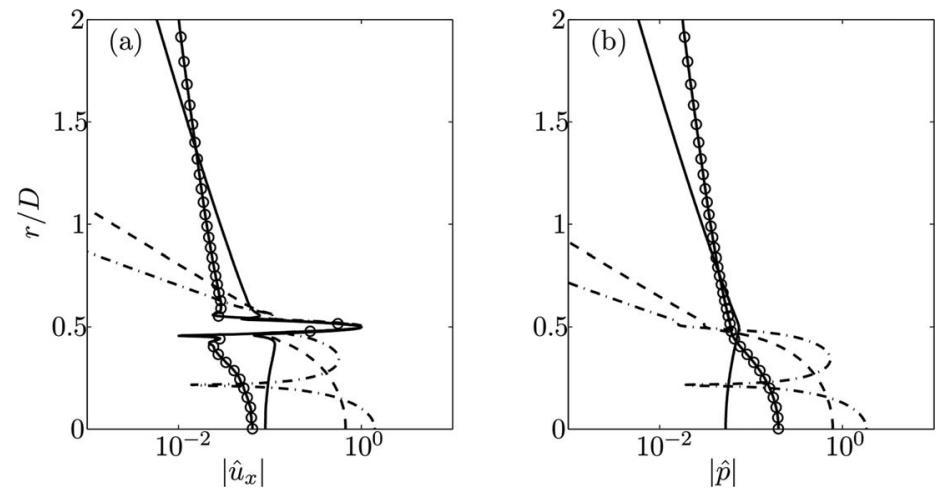

FIG. 2. Eigenfunctions of the linear instability waves for the isothermal $M_{j}=1.5$ jet at $x=0.5 D$, for $S t=0.3$ and $m=0$ : axial velocity (a) and pressure (b) components. Solid line with circles: $k=-1$; solid line: $k=0$; dashed line: $k=1$; dashed-dotted line: $k=2$.

have negative phase velocity, they correspond to downstream propagating waves; application of the Briggs criterion ${ }^{41}$ shows that the group velocity corresponding to these waves is positive. ${ }^{19}$

In the outer region, the eigenfunctions decay along the radial direction following the asymptotic decay rate for hydrodynamic waves in uniform flow $\sim r^{-1 / 2} \exp \left(-r \sqrt{\alpha^{2}-\omega^{2}}\right) .^{42}$ For a fixed frequency $\omega$, the decay rate increases with increasing number of peaks $k$, following the decrease in the phase velocity. Consequently, only the lower $k$ eigenmodes can contribute noticeably to the pressure fluctuations in the outer field. Eigenmode $k=-1$ is characterized by a supersonic negative phase velocity ( $\alpha_{r}$ is a small negative number), and accordingly its radial decay rate is the lowest among all instability waves: an important contribution to the near acoustic field may be associated with it. In contrast, Tam and $\mathrm{Hu}^{19}$ argued that states that the core pressure waves moving with supersonic phase velocity have negligible contribution to the pressure field outside of the annular mixing layer.

\section{B. Acoustic waves in the outer flow}

Inviscid instability analysis in the uniform outer flow reduces to the Helmholtz equation, the solutions of which define branches of acoustic waves. For a free-stream moving at subsonic velocity, the acoustic branches comprise a finite range of $\alpha$ corresponding to propagating waves and an infinite range of $\alpha$ corresponding to evanescent waves. The branch points, i.e., locations in the $\alpha$-plane where the branches commence, have phase velocity $u_{p h}=\omega / \alpha_{r}$, relative to the outer flow, equal to the speed of sound: $u_{p h}-\bar{u}_{c o}= \pm 1$ ( $\bar{u}_{c o}$ refers to the co-flow velocity). The propagating parts of the branch lie between the branch points and the turning point for which $\alpha=-\omega \bar{u}_{c o} /\left(1-\bar{u}_{c o}^{2}\right)$, and define neutral waves with real wavenumber $\alpha$. The evanescent part of the branches have phase velocity given by the turning point and extend to $\alpha_{i} \rightarrow \pm \infty$.

For a mean jet profile, the presence of the finite-thickness mixing layer blurs the distinction between propagating and evanescent waves, as the branches are no longer composed of straight lines, but describe curved lines. These branches define the grey cross and the filled squares in Figure 1. Note that the branch with $\alpha_{i}<0$ corresponds to decaying upstream-propagating waves. Figure 3 shows the axial velocity and pressure eigenfunctions corresponding to the four leading, downstreampropagating acoustic waves. The computational domain used for the computation of the eigenmodes in the figure extends up to $r=10 \mathrm{D}$. The discrete eigenmode recovered in the solution closest to the branch point presents a single maximum in the eigenfunctions in the outer flow. The number of peaks is increased by one as we move along the eigenvalues in the branch. Note that the acoustic waves exhibit a very slow radial decay compared to the hydrodynamic waves in Figure 2.

Two additional branches in the eigenspectra, denoted by open squares, are also classified as acoustic pressure waves. These branches are physically meaningful for $m \neq 0$. In the present computations the momentum conservation equation on the azimuthal direction is also maintained for $m=0$ permitting the appearance of these branches, but it is decoupled from the others and the 

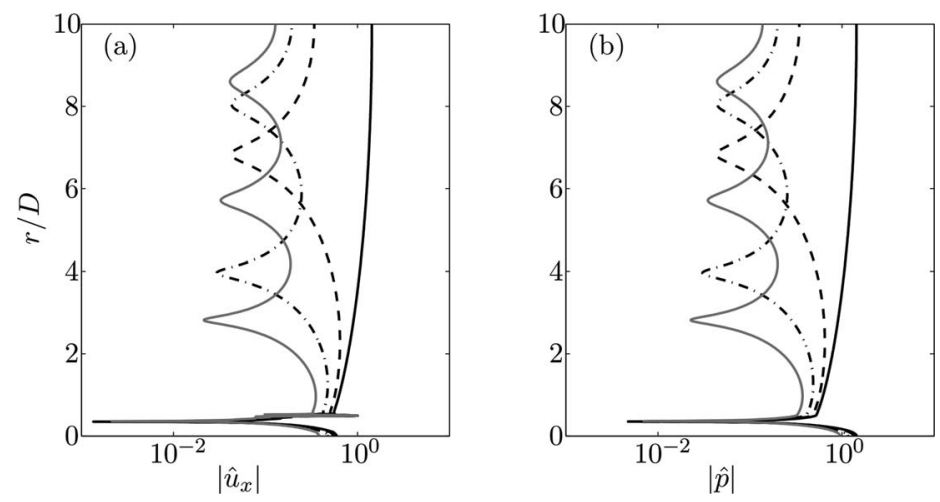

FIG. 3. Eigenfunctions of the acoustic waves for the isothermal $M_{j}=1.5$ jet at $x=0.5 D$ for $S t=0.3$ and $m=0$ : axial velocity (a) and pressure (b) components. The first 4 eigenmodes are shown.

branches correspond to spurious modes. These acoustic waves are evanescent and propagate at the speed of sound with respect to the outer flow. In the numerical solution, finite resolution distorts these branches towards higher phase velocities. However, these mathematical solutions are irrelevant for the present application.

\section{Vorticity and entropy waves}

The spatial framework of the linear stability theory for uniform base flow dictates the existence of two vorticity and two entropy wave branches. These waves are stable and propagate upstream and downstream with phase speed equal to the mean flow speed. The approximation of neglecting the second order axial derivative terms in the linear operators $\mathbf{L}$ and $\mathbf{R}$ neglects the upstream-propagating branches, as shown by $\mathrm{Li}$ and Malik, ${ }^{32}$ and consequently they are not recovered in the computed eigenspectrum (Figure 1, bottom).

Both the outer field and the potential core support vorticity and entropy waves, which are denoted in Figure 1 by vertical black solid and dotted lines, respectively. The phase velocities of these branches are equal to the mean flow in the respective spatial regions supporting them: $u_{p h}=\bar{u}_{c o}$ in the outer region and $u_{p h}=M_{\infty}$ in the core. Only small deviations from their theoretical uniform flow description appear as a consequence of the presence of the mixing layer, and the branches corresponding to vortical and entropic waves appear overlapped. For the present supersonic jet, the branches of core waves lie close to the K-H and pressure waves in the eigenspectrum. The mean speed in the outer region is small, so the associated wavenumbers are very high and lie outside of the window shown in the lower part of Figure 1. Like the pressure waves, the eigenmodes in the vorticity and entropy branches can be denumerated according to the number of peaks that the eigenfunctions present in the core or outer regions.

\section{EIGENMODE DECOMPOSITION OF SIMULATION DATA}

Time-dependent fluctuation data from the LES (discussed in Sec. II) are decomposed here into the contributions of the different eigenmode families. Following from the completeness of the LST eigenfunction system, the expansion of an arbitrary fluctuation profile will always deliver an accurate representation if a sufficient number of eigenmodes is used, but this convergence does not imply that the flow physics are linear. The bi-orthogonal projection is used here as a tool for the determination of the local contribution of some distinct physical mechanisms to the total fluctuation. In particular, three kinds of waves are of interest here: the dominant K-H instability, resonant pressure waves that may exist within the potential core, and the contribution of the acoustic branches. Branches of vorticity and entropy eigenmodes are considered to be unimportant in the determination of representative inlet conditions for the large-scale instability wave packets (as discussed in Sec. VI), 

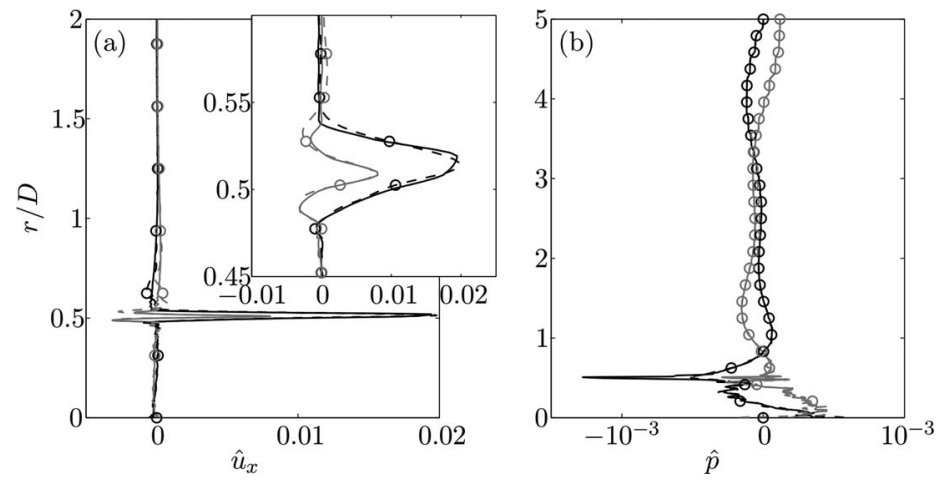

FIG. 4. Fluctuation profiles from LES (solid lines) and the projection on the first 501 LST eigenmodes (dashed lines with circles), for the isothermal $M_{j}=1.5$ jet at $x=0.5 D, S t=0.3$, and $m=0$. Black and grey lines correspond to the real and imaginary parts, respectively. Inset shows a zoom on the lipline. (a) $\hat{u}_{x}$ and (b) $\hat{p}$.

even though their contribution to the total fluctuation energy, especially in the mean shear region, may be significant.

Figure 4 compares the LES profiles of axial velocity and pressure for the isothermal jet, and the projection on a finite but large number of eigenmodes, for the Fourier mode $S t=0.3$ and $m=0$ at $x=0.5 D$, illustrating the convergence of the eigenmode expansion. As explained in Sec. III, if adjoint eigenfunctions were not adequately computed, the eigenmode expansion of an arbitrary fluctuation profile would not converge. A linear plot is used here in order to show the match in phase. The K-H eigenmode and the 500 eigenmodes closest to it are used in the projection. Note that only a few members of the vorticity and entropy waves in the outer region are included, as they present large $\alpha_{r}$ and lie far from the K-H eigenmode. This results in small differences between the LES and the projected profiles towards the outer part of the mixing layer, where the contribution of vortical eigenmodes is expected to be more relevant.

Figures 5-8 show the separate projections of the isothermal jet LES fluctuation profile on the $\mathrm{K}-\mathrm{H}$ eigenmode, the first pair $(k= \pm 1)$ of core pressure modes and the acoustic branches, compared to the original LES profile for different $(S t, m)$ modes.

The K-H eigenmode is found to be the dominant contributor to the lipline velocity fluctuations in most of the Fourier modes examined. While it is customarily assumed that the K-H eigenmode is in fact responsible for nearly all the fluctuations at the lipline, the present results show that many other eigenmodes can contribute in a non-negligible manner to the total fluctuations in the near-nozzle cross sections. The pressure component of the $\mathrm{K}-\mathrm{H}$ eigenmode is also found to be significant in the
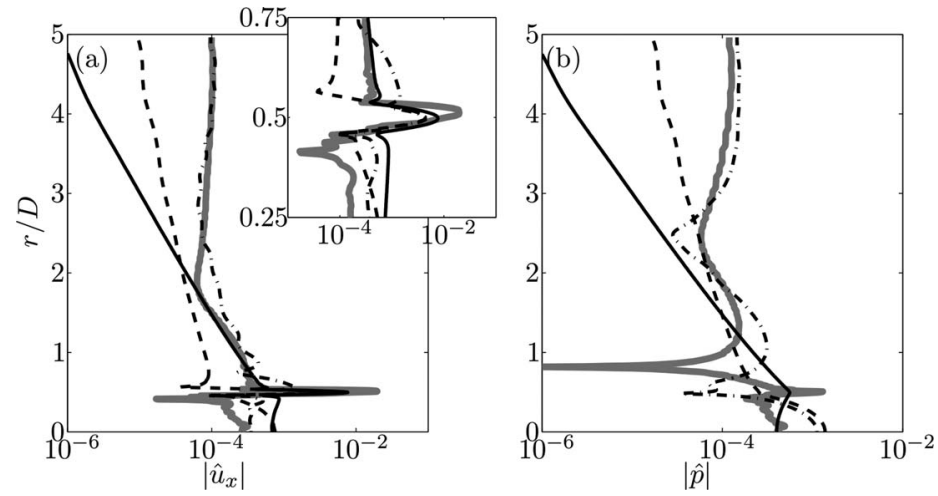

FIG. 5. Fluctuation profiles from LES (thick grey lines), and their projection on the Kelvin-Helmholtz eigenmode (solid black lines), core pressure modes $k= \pm 1$ (dashed black lines), and acoustic families (dotted-dashed black lines). Isothermal jet at $S t=0.3, m=0$, and $x=0.5 D$. Inset shows a zoom on the lipline. (a) $\left|\hat{u}_{x}\right|$ and (b) $|\hat{p}|$. 

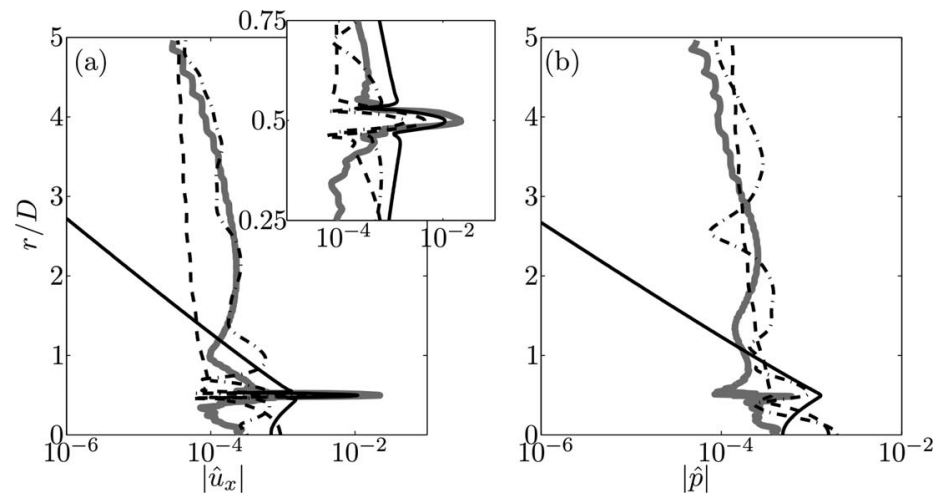

FIG. 6. Fluctuation profiles from LES (thick grey lines), and their projection on the Kelvin-Helmholtz eigenmode (solid black lines), core pressure modes $k= \pm 1$ (dashed black lines), and acoustic families (dotted-dashed black lines). Isothermal jet at $S t=0.5, m=0$, and $x=0.5 D$. Inset shows a zoom on the lipline. (a) $\left|\hat{u}_{x}\right|$ and (b) $|\hat{p}|$.

vicinity of the lipline, but its fast decay in the radial direction makes its direct contribution to the outer field of less relevance.

Attention is turned now to the resonant pressure waves in the potential core. Only the first pair $k$ $= \pm 1$ is used in the eigenmode projections shown here. The following pairs $(k= \pm 2, \pm 3, \ldots)$ were found to have much smaller amplitudes and to decay faster in the radial direction. The amplitudes attained by these eigenmodes inside of the potential core surpass those of the LES profiles, but they cancel out with the contributions of other core pressure modes, and the core vorticity and entropy waves. The eigenmode $k=-1$ has, in the range of frequencies and azimuthal wavenumbers studied, supersonic phase velocity. The radial decay of the eigenfunction, though governed by the hydrodynamic asymptotic behavior, is very small and the eigenmode contributes to the velocity and pressure field in the outer flow in a significant manner over the first few diameters from the jet axis. However, the axial decay rate of this eigenmode is high. The physical meaning of this particular eigenmode is unclear: the parallel-flow approximation leading to the LST that describes them ${ }^{19}$ does not take into account the diverging nature of the real jet and the closure of the potential core. The appearance of the cross-hatched patterns observed experimentally ${ }^{20}$ was explained by the existence of resonant pressure waves inside an infinite potential core. Relating that interpretation to the present results does not seem to be straightforward, as the wavelengths associated with the $k=-1$ eigenmode are of the same order of magnitude as the actual axial extent of the mean potential core (between 6 and 7 diameters), invalidating the assumptions in LST. A recent study ${ }^{14}$ using a global (multi-dimensional) eigenvalue problem recovered resonant pressure waves inside of
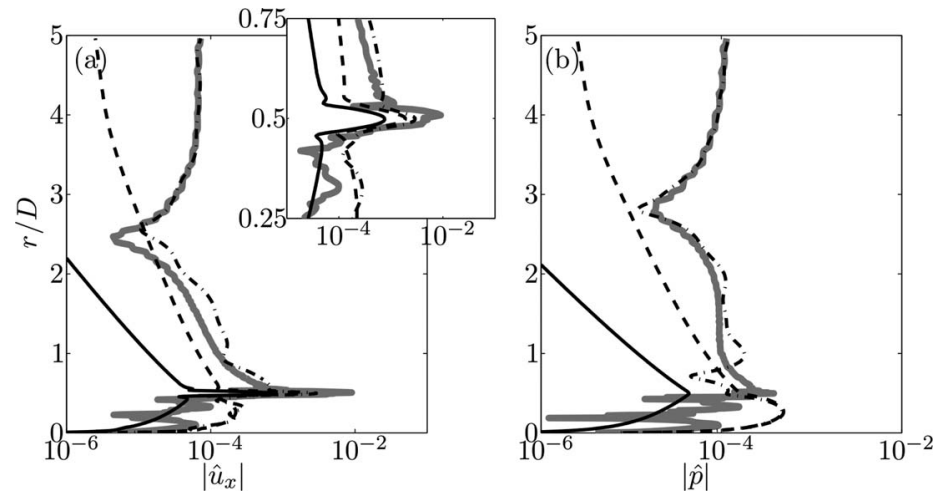

FIG. 7. Fluctuation profiles from LES (thick grey lines), and their projection on the Kelvin-Helmholtz eigenmode (solid black lines), core pressure modes $k= \pm 1$ (dashed black lines), and acoustic families (dotted-dashed black lines). Isothermal jet at $S t=0.3, m=1$, and $x=0.5 D$. Inset shows a zoom on the lipline. (a) $\left|\hat{u}_{x}\right|$ and (b) $|\hat{p}|$. 

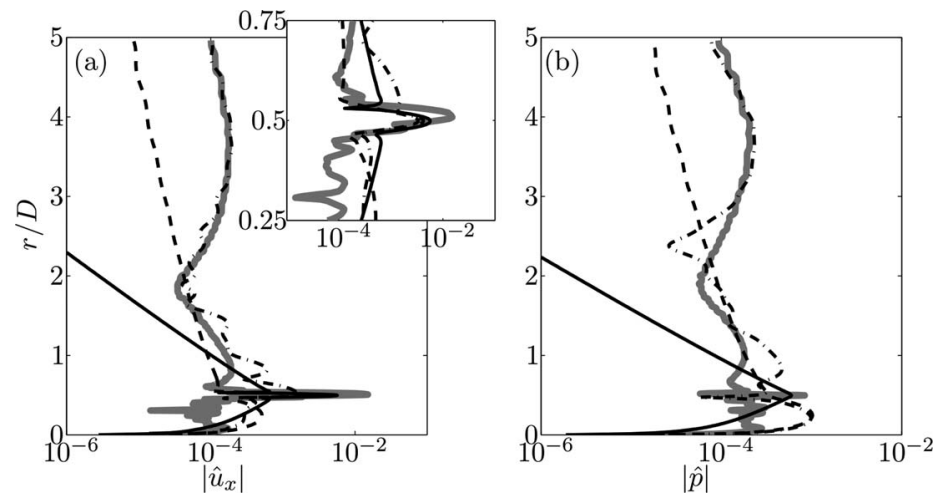

FIG. 8. Fluctuation profiles from LES (thick grey lines), and their projection on the Kelvin-Helmholtz eigenmode (solid black lines), core pressure modes $k= \pm 1$ (dashed black lines), and acoustic families (dotted-dashed black lines). Isothermal jet at $S t=0.5, m=1$, and $x=0.5 D$. Inset shows a zoom on the lipline. (a) $\left|\hat{u}_{x}\right|$ and (b) $|\hat{p}|$.

the finite-length potential core; their perturbation shape functions do not satisfy the quasi-parallel approximation indicating that neither LST nor PSE are adequate tools for their study, a more general approach being necessary. However, the resonant core pressure-wave eigenmodes found by Nichols and Lele ${ }^{14}$ exhibit a clear noise radiation pattern emerging from the nozzle lip and propagating predominantly in the upstream direction, in line with the dominance of the $k=-1$ eigenmode found in the present projections.

The contributions of a relatively small number of eigenmodes belonging to the upstream and downstream-propagating branches of acoustic waves are shown together in Figures 5-8. The number of discrete eigenmodes that need to be considered for the convergence of the projection depends on the radial extent of the truncated computational domain. In the present results the projected profiles are visually indistinguishable when more than 20 eigenmodes in each branch are introduced. The 20th eigenmode has axial damping rate $\alpha_{i} \approx 15$ and thus decays several orders of magnitude in one jet diameter, so its relative importance in the total acoustic field is very small. The projected acoustic field represents accurately the LES perturbation profiles at the outer domain boundary, and the agreement is maintained until the jet axis in those cases in which the amplitude of the core pressure modes is comparatively small. This not only confirms that the outer-field vortical and entropic waves are practically non-existent in the surroundings of the nozzle lip, as should be expected from the simulation set up, but also attests to the presence of upstream-propagating acoustic waves. The separate projection on the upstream-propagating acoustic waves (not shown in the figures) attains much higher amplitudes than those corresponding to the downstream-propagating ones. This acoustic radiation must be generated by downstream turbulence and is, therefore, not relevant to the determination of the inlet conditions for the wave packet computation.

The application of the eigenmode decomposition to the hot jet data delivers qualitatively identical results, and therefore are not reproduced here.

\section{INLET CONDITIONS AND WAVE PACKETS}

The eigenmode decomposition of LES fluctuations is now used to determine inlet conditions for PSE calculations of the wave packets, by assigning the correct amplitude and phase to each eigenmode of interest and filtering out the other fluctuations. These inlet conditions could in principle be used directly in linearized Navier-Stokes ${ }^{15,17}$ or Euler simulations, ${ }^{12}$ or along with global eigenvalue problems ${ }^{14}$ in order to compute calibrated wave packets. On the other hand, when the PSE ansatz (2) is imposed, the resulting instability wave is completely defined by a single slowly varying wavenumber $\alpha(x)$ and one shape function $\tilde{\mathbf{q}}(x, r)$ for each $(S t, m)$ mode. Consequently, PSE is unable to retain much of the complexity of the imposed inlet fluctuation. The downstream integration of the parabolized equations tends to converge to the most unstable local eigenmode 
(a)

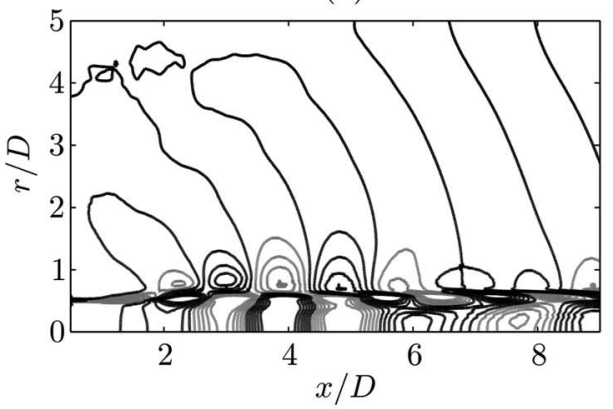

(c)

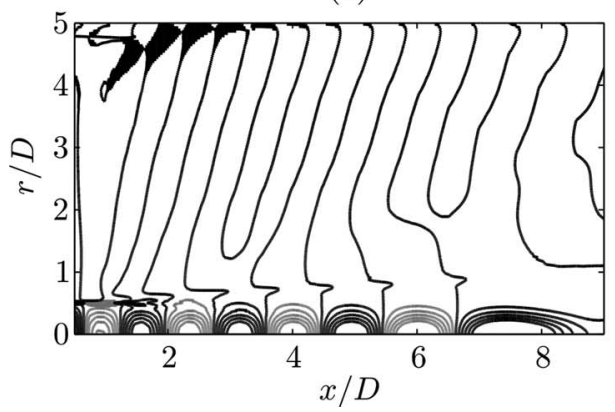

(b)

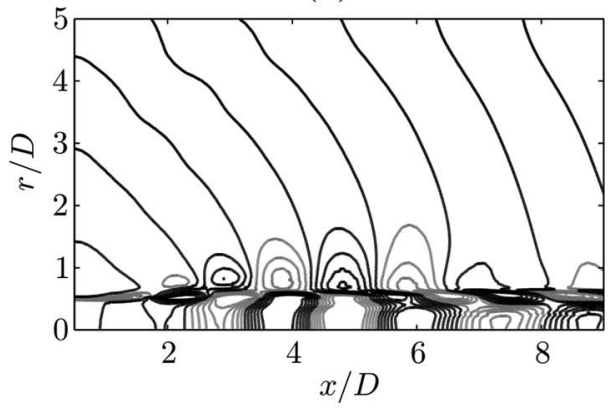

(d)

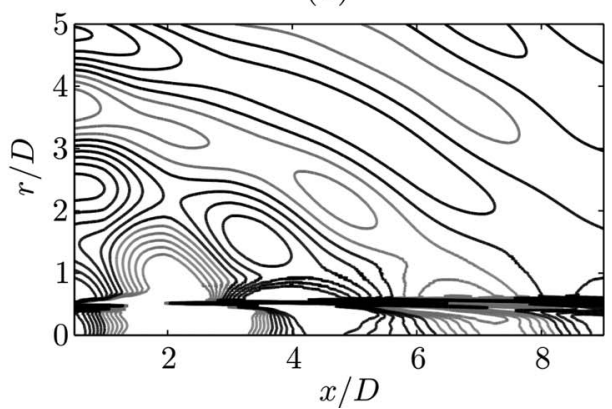

FIG. 9. Axial velocity component of the PSE solutions for the isothermal jet at $S t=0.3$ and $m=0$, with different inlet conditions at $x=0.5 D$. (a) LES profile; contours: $-10^{-2}\left(10^{-3}\right) 10^{-2}$. (b) K-H eigenmode; contours: $-10^{-2}\left(10^{-3}\right) 10^{-2}$. (c) Resonant core eigenmode $k=1$; contours: $-5 \times 10^{-4}\left(10^{-4}\right) 5 \times 10^{-4}$. (d) First five downstream propagating acoustic eigenmodes; contours: $-2.5 \times 10^{-5}\left(5 \times 10^{-6}\right) 2.5 \times 10^{-5}$. Grey lines correspond to negative contours, black lines correspond to $u_{x}=0$ and positive contours.

in the proximity of the solution computed at the previous axial location, or to the imposed inlet conditions at the first axial step. ${ }^{32}$

This is not necessarily a drawback in the computation of the large-scale wave packets in ideally expanded turbulent jets, as the inflectional instability is the only physical mechanism that can give rise by itself to convectively unstable hydrodynamic perturbations coherent over significant axial extents. The presence of systems of resonant pressure waves within the potential core is a possible noise source, as discussed in Sec. V, but its relative importance is expected to be small for the present simulations of ideally expanded jets..$^{29}$ On the other hand, PSE is not adequate for the direct integration of the acoustic branches as the solution will converge towards the branch points and the contribution of most of the branch will be artificially damped by the integration algorithm. Finally, the upstream-propagating eigenmodes, that are neglected by the PSE approximation and the PSE-based LST approach used in this work, are unrelated to the wave packets, which are advected downstream.

Figure 9 shows the PSE solutions corresponding to different inlet conditions, for the same $S t=0.3$ and $m=0$ mode. The axial velocity component is displayed in the comparison in order to illustrate the fine spatial structure of the fluctuations near the lipline. The isothermal jet is considered and the inlet section is located at $x=0.5 D$, but the results do not change significantly for inlet sections between $0.25 D$ and $1 D .^{43}$ Four inlet conditions are considered: case (a) imposes the perturbation profile as obtained directly from the DFT of a single time-segment of the LES data, without projecting on the eigenmodes; case (b) uses the projection of the above-mentioned LES profile on the K-H eigenmode alone; case (c) is initialized with the contribution of the core pressure eigenmode $k=1$; and case (d) imposes the projection of the first five eigenmodes in the downstream propagating branch of acoustic modes. The inlet wavenumber is set to $\alpha\left(x_{0}\right)=0$ in all cases. The iterative process in the PSE solution adapts the value of $\alpha$ according to the perturbation profile, so that in the first solution step it is close to that of the leading eigenmode present in $\tilde{\mathbf{q}}\left(x_{0}\right)$. 
$S t=0.1$
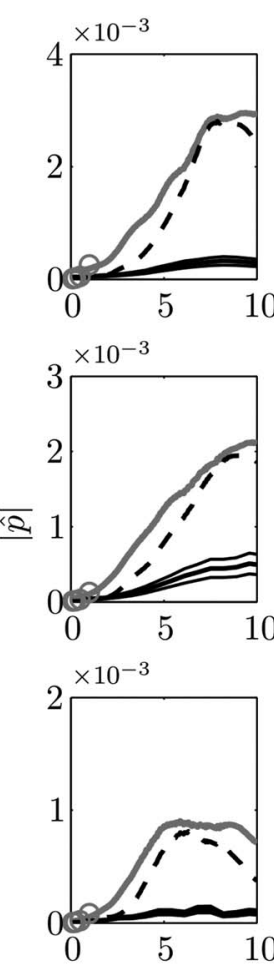

$$
S t=0.2
$$

$S t=0.3$

$$
S t=0.4
$$$$
S t=0.5
$$
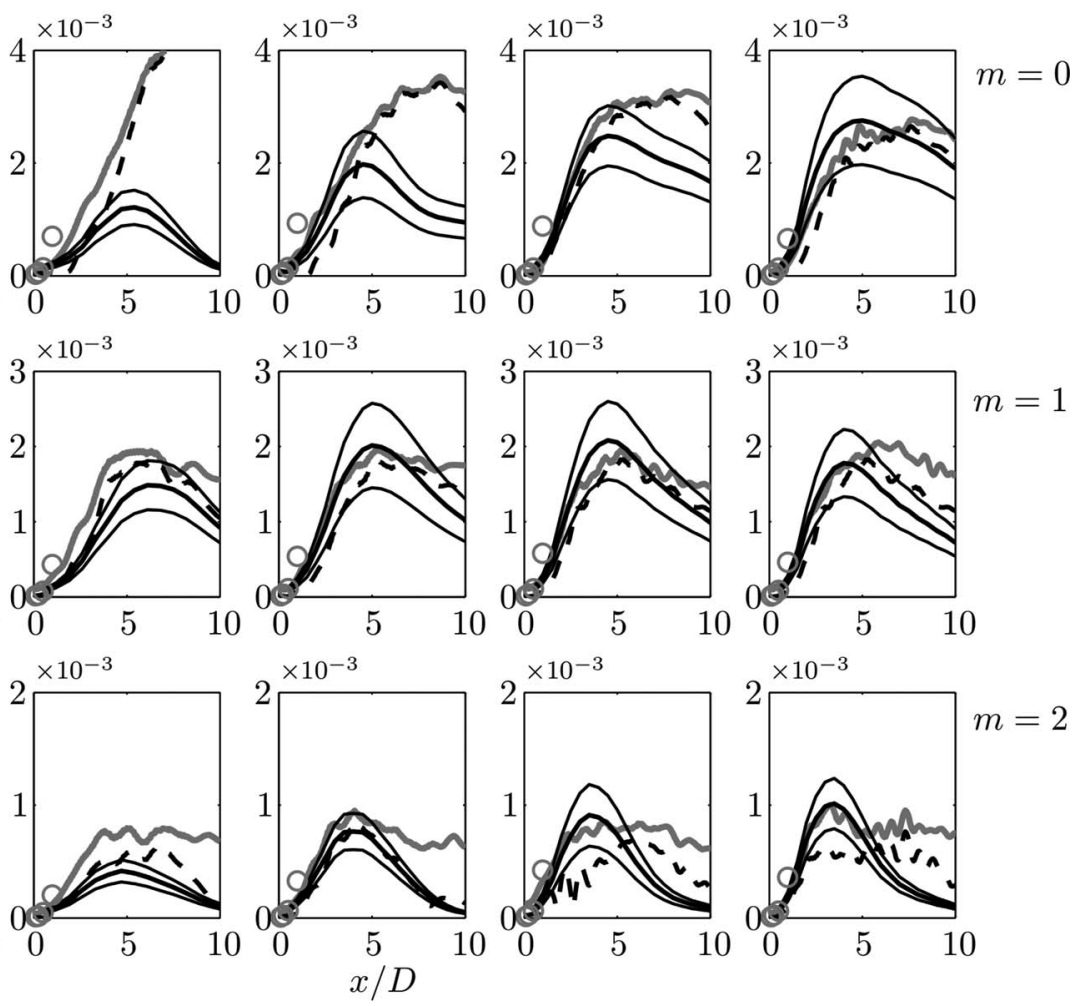

FIG. 10. Pressure amplitude envelope at the near-field cone for the isothermal $M_{j}=1.5$ jet. LES data (thick grey lines); first POD mode (dashed black lines); average of PSE wave packets (thick solid black lines); average \pm 0.5 standard deviation of PSE wave packets (thin solid black lines); LES data projected on the K-H eigenmode (grey circles).

Note the different contour scales used in the figure for the different cases. The PSE integration yields very similar results when the LES data are directly used as inlet conditions compared to when it is first projected onto the K-H eigenmode, both in terms of perturbation shapes and amplitudes. On the other hand, the spatial structure of the PSE solution differs remarkably when eigenmode contributions other than that of the K-H eigenmode are considered. In addition, the perturbation amplitudes corresponding to these solutions are substantially lower than those of cases (a) and (b). These observations justify the use of the K-H eigenmode, previously calibrated in amplitude via the adjoint projection of the LES data, as inlet conditions in the PSE modeling of wave packets in ideally expanded supersonic jets.

The LES data used in the eigenmode decomposition comprise the DFT of a single time-segment for each projection, but the perturbation profiles corresponding to different segments may differ notably along the total simulation time, having a strong impact on the projected amplitudes $a_{n}$. Using the LES profile averaged over segments is not correct as the phase relations are not adequately preserved in the averaging process. In order to compare PSE wave packets with their counterparts on the LES data, each time-segment is considered as an individual realization of the flow field. For each segment, the DFT's profile is projected on the K-H eigenmode for every $(S t, m)$ mode and used as inlet condition in the computations. The solution of linear PSE is independent of the initial amplitude and phase, so that a single PSE wave packet needs to be computed and then rescaled using the projection amplitude for each segment. The resulting PSE wave packets are then averaged. The standard deviation of the PSE solutions is also computed to illustrate the variability of the wave packets across segments.

In order to streamline comparisons between the LES data and PSE results, the pressure component is extracted at a virtual cone representative of the position of an experimental microphone 


$$
S t=0.1
$$

$$
S t=0.2
$$$$
S t=0.3
$$$$
S t=0.4
$$$$
S t=0.5
$$
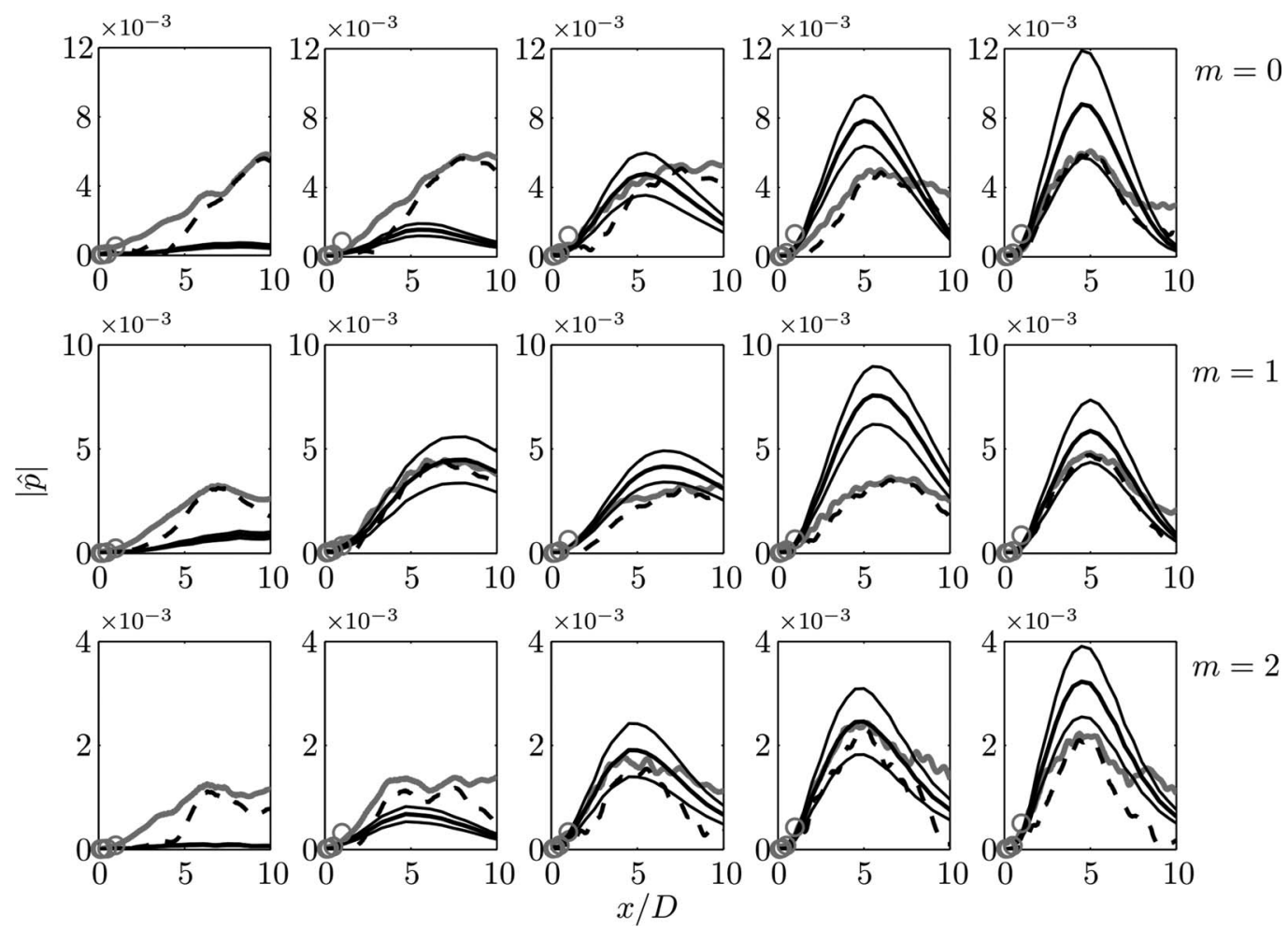

FIG. 11. Pressure amplitude envelope at the near-field cone for the hot $M_{j}=1.5$ jet. LES data (thick grey lines); first POD mode (dashed black lines); average of PSE wave packets (thick solid black lines); average \pm 0.5 standard deviation of PSE wave packets (thin solid black lines); LES data projected on the K-H eigenmode (grey circles).

array. ${ }^{28}$ The cone is located immediately outside of the turbulent mixing region, where the hydrodynamic component of the pressure is expected to dominate. ${ }^{27}$ Analogous comparisons showed very good agreement between PSE and experiments for subsonic jets, ${ }^{13}$ especially in the first diameters of axial development up to the peak in pressure amplitude. The comparison with the averaged pressure became poorer downstream of the peak, as the measurements contained other contributions, presumably of acoustic nature, unrelated to the wave packets. A POD was used to educe the pressure contributions with the strongest coherence over the entire microphone array, improving significantly the comparisons with the PSE computations.

Analogous comparisons are presented here. Pressure amplitude distributions are computed from the LES data as the square-root of the power spectral density using the same DFT parameters as for the inlet profiles (see Sec. II). In addition, POD-based large-scale structures eduction is performed on the LES data considering cross-correlations of the pressure along the virtual cone, following the procedure described in Gudmundsson and Colonius. ${ }^{13}$

Figure 10 compares the PSE results with the averaged and first POD mode of the LES data for the isothermal jet, at different $(S t, m)$ modes. In line with related results reported in the literature, ${ }^{3,44}$ the agreement is poor for the lowest $(S t \leq 0.2)$ frequencies. The disagreement may be attributed to an important influence of nonlinear interactions on these modes ${ }^{17}$ or to the inadequacy of the PSE approach for the treatment of low frequencies, as their axial wavelengths become comparable to the length of the potential core itself, thus violating the separation of scales. On the other hand, remarkable agreement with the averaged LES data is found from the inlet location up to the location of the peak amplitude for $S t \geq 0.3$. Past the amplitude peak, the PSE and raw LES data diverge in the same manner as was observed for subsonic jets. Considering the first POD mode in this region improves the comparison, but to a rather modest degree compared with the excellent comparisons attained for the unheated subsonic jets. ${ }^{13}$ However, this may be expected for supersonic jets on 
account of the much stronger acoustic field that can require multiple orthogonal POD modes in order to reproduce adequately the near-field pressure fluctuations. ${ }^{43}$

Comparisons for the hot jet are shown in Figure 11. Similar observations to those for the isothermal jet can be made here. For the hot jet, the solution of the PSE computations initialized with the projected K-H eigenmode tends to overestimate the peak amplitude at the higher frequencies.

The results presented in Figures 10 and 11 correspond to PSE solutions with amplitudes determined from the projection of LES data at the section $x_{0}=0.5 \mathrm{D}$. As stated before, the PSE wave packet models should be consistent in spatial shape and amplitude if the inlet location is chosen at any cross-section close enough to the nozzle lip. The grey circles in Figures 10 and 11 correspond to the bi-orthogonal projection of the LES data on the K-H eigenmode at the axial sections $x_{0}=0.25,0.5,0.75$, and $1 D$. Comparing these amplitudes with the baseline PSE solution initiated at $x_{0}=0.5 D$, it demonstrates that the computed wave packet amplitudes are consistent if computed in the range $0 \leq x_{0} \leq 0.75 D$. At $x_{0}=1 D$ the K-H amplitude computed from the projection clearly exceeds the amplitude of the LES solution itself, serving as an indication of the relevance of other eigenmode families in the projection. From a physical point of view, the reason why the bi-orthogonal projection is no longer useful for $x_{0} \geq 1 D$ is related to the transitional nature of the annular shear-layer. As no tripping exists inside the nozzle, the shear-layer is initially laminar and the growth of the disturbances is likely to be linear for most of the frequency range of interest. In this case, the bi-orthogonal projection is theoretically well-founded. Between $0.75 D$ and $1 D$ downstream of the nozzle lip the shear-layer becomes of transitional nature and the projection does not deliver consistent results for the wave packet amplitude determination.

\section{CONCLUSIONS}

Linear parabolized stability equations are a useful tool for the computation of instability wave packets modeling the statistical behavior of the large-scale structures in turbulent jets. Previous experience with subsonic jets showed excellent agreement between the near-field pressure envelope computed by PSE and phased-array measurements, ${ }^{13}$ and between the computed velocity field and the one measured using time-resolved particle image velocimetry. ${ }^{3}$ There, the amplitude calibration of the linear PSE wave packets was done by fitting directly with the experimental values. Two main reasons justify this approach. First, LST for subsonic jets shows that the only possible modal instability is the Kelvin-Helmholtz mechanism, which is convectively unstable except for high temperature and Mach number jets. Second, the subsonic Mach number implies that the acoustic component of the pressure near-field is much weaker than the hydrodynamic part corresponding to the instability wave. Consequently, in the absence of additional disturbance sources (e.g., perturbations in the potential core originated upstream of the nozzle lip) most of the fluctuations in the first diameters downstream of the nozzle are associated with the K-H instability. This is not necessarily the case for supersonic jets, for which linear stability theory predicts the existence of additional instability mechanisms and a much stronger acoustic field is expected.

The present investigation considered two ideally expanded jets, one isothermal and one moderately heated, for which a high-quality LES database was available. In the simulations, a long time sample was used in order to achieve reasonable statistical convergence of the low frequencies, and a relatively fine spatial resolution was used in the vicinity of the nozzle lip to reproduce accurately the initial shear layer. The quality of the LES database is instrumental in the determination of the inlet conditions, as well in the subsequent comparisons between the modeled wave packets and those existing in the turbulent flow.

The local, spatial linear stability eigenvalue problem under the parallel-flow approximation was used to derive an eigenmode decomposition technique that permits the projection of the local fluctuation profiles from LES on different subsets of eigenmodes. These projections showed that the $\mathrm{K}-\mathrm{H}$ eigenmode, while being dominant for most of the frequencies and azimuthal wavenumbers of interest, was only responsible for a fraction of the fluctuations. Additionally, the acoustic branches and a family of pressure waves resonant within the potential core were found to be of relevance in the LES fluctuation profiles close to the lip. The core pressure waves from the local stability analysis are suggested to be related to a global instability mechanism ${ }^{14}$ that radiates sound in the 
upstream direction. It was also found that most of the acoustic field near the nozzle lip section was constituted of upstream-propagating waves. These findings, along with the particularities of the PSE ansatz, suggest that only the contribution of the K-H eigenmode need to be included in the inlet conditions, as was the case for the subsonic jets discussed above. The averaged PSE wave packets computed using the projection of different time segments of the LES data on the K-H eigenmode as the inlet condition was found to be in very good agreement with the LES pressure near-field up to the location of the amplitude peak, for the range of frequencies $S t=0.3-0.5$ and azimuthal wavenumbers $m=0,1$, and 2 . An important variability of the wave packet amplitudes, related to the temporal intermittency observed in experiments, ${ }^{45,46}$ is also observed in the present results.

Knowledge of the initial K-H amplitude on a statistical basis can be used to calibrate the linear wave packets. The determination of the initial amplitudes is a receptivity problem that requires of detailed information of the disturbances existing upstream of the nozzle lip and the state of the inner boundary layer, and their dependency on the jet parameters such as the jet exhaust Mach number and temperature ratio. The convective nature of the inflectional instability that gives rise to the wave packets implies that once the amplitudes at a single near-nozzle cross-section are determined, the PSE models predict the axial evolution of the wave packet amplitudes without further information. Experimental or high-fidelity simulation data (like the present LES database) of the upstream and near-nozzle flow can be used to determine correlations between these parameters and the inlet perturbations to be used in the calibration of wave packet models for engineering purposes.

\section{ACKNOWLEDGMENTS}

This work was sponsored in part by the U.S. Navy Naval Air Systems Command (Contract No. N68335-11-C-0026) and by the Office of Naval Research (Grant No. N0014-11-1-0753). Any opinions, findings, and conclusions or recommendations expressed in this material are those of the author(s) and do not necessarily reflect the views of the sponsoring agencies. We would also like to thank Dr. Joseph Nichols from Stanford University, Center for Turbulent Research, for his many helpful comments on this work. D. Rodríguez acknowledges funding from the Marie Curie - COFUND-UNITE programme. The authors thank the anonymous referees for their valuable comments that improved the quality of the paper.

\section{APPENDIX A: NUMERICAL IMPLEMENTATION}

The linear operators conforming both the LST and PSE problems are discretized using fourthorder central finite differences in the radial direction, with an appropriate mapping in order to cluster points in the lipline region. The outer boundary of the domain is closed with the characteristic boundary conditions of Thompson. ${ }^{47}$ The boundary conditions at the centerline are derived following Mohseni and Colonius. ${ }^{48}$ The discretized versions of the linear operators $\mathbf{A}$ through $\mathbf{E}$ are stored in sparse format, exploiting the banded structure of the differentiation matrix.

In the discretization of the operators, resolutions larger than those usual in PSE computations are used. It is found that 200-300 discretization points suffice in order to converge the K-H eigenmode, but the convergence of the eigenmode decomposition required 950 points on the radial direction. A maximum of 1500 points was used in order to evaluate the convergence. These high resolutions are required due to the use of the discrete adjoint: an unphysical behavior appears near the outer boundary in the adjoint eigenfunctions due to the characteristic boundary conditions that is reduced as the resolution increases. The eigenfunctions corresponding to acoustic modes are especially sensitive to resolution. The bi-orthogonal projections shown in Figures $4-8$ are computed in a domain extending up to $r=5 D$. The acoustic eigenfunctions in Figure 3 were computed in a larger domain to illustrate clearly the oscillations in the outer flow.

Two different methods were implemented for the solution of the eigenvalue problems (6) and (7). The first method stores temporally the matrices $\mathbf{L}$ and $\mathbf{R}$ in dense format, and uses the QZ algorithm implementation (routine ZGEEV) included in the open library Linear Algebra PACKage (LAPACK) for the solution of generalized eigenvalue problems of non-symmetric complex matrices. This approach permits computing the right and left eigenvectors of the problem at the same time, thus 
solving both the direct and adjoint problems simultaneously. However, the dense storage demands an order of magnitude more memory than the sparse approach, and becomes prohibitive in terms of central processing unit (CPU) time for the resolutions employed here. The second method for the solution of the EVP is an sparse implementation of the Arnoldi's algorithm ${ }^{49,50}$ that permits recovering a window of the eigenspectrum by means of an iterative method. The sparse linear algebra package MUMPS ${ }^{51}$ is employed in the solution of the associated linear problems. A shift-and-invert transformation is used, transforming the problem to be solved into

$$
(\mathbf{R}-\mathrm{i} \sigma \mathbf{L})^{-1} \mathbf{L} \hat{\mathbf{q}}=\mu \hat{\mathbf{q}},
$$

where $\mu=-\mathrm{i}(\alpha-\sigma)^{-1}$. The shift parameter $\sigma$ is used to control the center of the eigenvalue window: the eigenvalues closer to $\sigma$ will be the first to converge. The present implementation of the Arnoldi's algorithm does not permit the direct computation of the left eigenvectors, and (7) must be solved explicitly. The results delivered by the two different EVP solvers were cross-checked, obtaining identical results for the converged eigenvalues.

Parabolized stability equations (4) are integrated along the axial direction using an implicit Euler scheme, in which the solution $\tilde{\mathbf{q}}_{j+1}$ at the axial step $x_{j+1}$ is obtained from $\tilde{\mathbf{q}}_{j}$ by solving

$$
\mathbf{L}_{j+1}\left(\frac{\tilde{\mathbf{q}}_{j+1}-\tilde{\mathbf{q}}_{j}}{\Delta x}\right)=\mathbf{R}_{j+1} \tilde{\mathbf{q}}_{j+1}
$$

where $\Delta x=x_{j+1}-x_{j}$. Note that $\mathbf{L}$ and $\mathbf{R}$ depend on the mean flow quantities, and hence their dependence on the axial location. The solution of (A2) is computed using the sparse library MUMPS.

\section{APPENDIX B: RELATION BETWEEN THE MATRIX OPERATORS OF PSE AND LST}

The homogeneous linearized Navier-Stokes equations, after Fourier transform in time and azimuthal direction, can be written as

$$
\left(\mathbf{H}_{0}+\mathbf{H}_{x} \frac{\partial}{\partial x}+\mathbf{H}_{x x} \frac{\partial^{2}}{\partial x^{2}}+\mathbf{H}_{r} \frac{\partial}{\partial r}+\mathbf{H}_{x r} \frac{\partial^{2}}{\partial x r}+\mathbf{H}_{r r} \frac{\partial^{2}}{\partial r^{2}}\right) \breve{\mathbf{q}}_{\omega, m}=0 .
$$

Upon introduction of the PSE ansatz (2) into (B1), one arrives at the homogeneous form of Eq. (3) by defining the operators:

$$
\begin{aligned}
& \mathbf{A}=\mathbf{H}_{0}+\mathrm{i} \alpha \mathbf{H}_{\mathrm{x}}-\alpha^{2} \mathbf{H}_{\mathrm{xx}}, \\
& \mathbf{B}=\mathrm{i} \mathbf{H}_{x x}, \\
& \mathbf{C}=\mathbf{H}_{x}+2 \mathrm{i} \alpha \mathbf{H}_{x x}, \\
& \mathbf{D}=\mathbf{H}_{r}+\mathrm{i} \alpha \mathbf{H}_{x r}, \\
& \mathbf{E}=\mathbf{H}_{r r} \\
& \mathbf{F}=\mathbf{H}_{x r},
\end{aligned}
$$

so that $\mathbf{L}$ and $\mathbf{R}$ in Eq. (4) take the form

$$
\begin{aligned}
& \mathbf{L}=\mathbf{H}_{x}+2 \mathrm{i} \alpha \mathbf{H}_{x x}+\mathbf{H}_{x r} \frac{\partial}{\partial r}, \\
& \mathbf{R}=-\left(\mathbf{H}_{0}+\mathrm{i} \alpha \mathbf{H}_{x}-\alpha^{2} \mathbf{H}_{x x}+\mathbf{H}_{r} \frac{\partial}{\partial r}+\mathrm{i} \alpha \mathbf{H}_{x r} \frac{\partial}{\partial r}+\mathbf{H}_{r r} \frac{\partial^{2}}{\partial r^{2}}\right) .
\end{aligned}
$$


On the other hand, introducing in Eq. (B1) the LST ansatz $\breve{\mathbf{q}}_{\omega, m}(x, r)=\hat{\mathbf{q}}_{\omega, m}(r) \mathrm{e}^{\mathrm{i} \alpha x}$, results

$$
\left(\mathbf{H}_{0}+\mathrm{i} \alpha \mathbf{H}_{x}-\alpha^{2} \mathbf{H}_{x x}+\mathbf{H}_{r} \frac{\partial}{\partial r}+\mathrm{i} \alpha \mathbf{H}_{x r} \frac{\partial}{\partial r}+\mathbf{H}_{r r} \frac{\partial^{2}}{\partial r^{2}}\right) \hat{\mathbf{q}}_{\omega, m}=0 .
$$

Neglecting $\alpha^{2}$ terms and recasting as a linear EVP for $\alpha$ :

$$
\mathrm{i} \alpha\left(\mathbf{H}_{x}+\mathbf{H}_{x r} \frac{\partial}{\partial r}\right) \hat{\mathbf{q}}_{\omega, m}=\left(-\mathbf{H}_{0}-\mathbf{H}_{r} \frac{\partial}{\partial r}-\mathbf{H}_{r r} \frac{\partial^{2}}{\partial r^{2}}\right) \hat{\mathbf{q}}_{\omega, m} .
$$

When particularized for $\alpha=0$, the matrices $\mathbf{L}$ in (B8) and $\mathbf{R}$ in (B9) are, respectively, identical to the left- and right-hand side matrices in Eq. (B11).

${ }^{1}$ P. Jordan and T. Colonius, "Wave packets and turbulent jet noise," Annu. Rev. Fluid Mech. 45, 173-195 (2013).

${ }^{2}$ A. Michalke and H. V. Fuchs, "On turbulence and noise of an axisymmetric shear flow," J. Fluid Mech. 70, 179-205 (1975).

${ }^{3}$ A. Cavalieri, D. Rodríguez, P. Jordan, T. Colonius, and Y. Gervais, "Wavepackets in the velocity field of turbulent jets," J. Fluid Mech. 730, 559-592 (2013).

${ }^{4}$ D. G. Crighton and M. Gaster, "Stability of slowly diverging jet flow,” J. Fluid Mech. 77(2), 397-413 (1976).

${ }^{5}$ R. Mankbadi and J. T. C. Liu, "A study of the interactions between large-scale coherent structures and fine-grained turbulence in a round jet," Proc. R. Soc. London 1443, 541-602 (1981).

${ }^{6}$ C. Tam, "Supersonic jet noise generated by large scale disturbances," J. Sound Vib. 38(1), 51-79 (1975).

${ }^{7}$ P. Ray, L. Cheung, and S. Lele, "On the growth and propagation of linear instability waves in compressible turbulent jets," Phys. Fluids 21, 054106 (2009).

${ }^{8}$ A. Michalke, "Survey on jet instability theory," Prog. Aeronaut. Sci. 21, 159-199 (1984).

${ }^{9}$ C. Tam and P. Morris, "Tone excited jets - Part V: A theoretical model and comparison with experiments," J. Sound Vib. 102, 119-151 (1985).

${ }^{10}$ P. Balakumar, "Prediction of supersonic jet noise," AIAA Paper 98-1057, 1998.

${ }^{11}$ M. R. Malik and C. L. Chang, "Nonparallel and nonlinear stability of supersonic jet flow," Comput. Fluids 29, 327-365 (2000).

${ }^{12}$ E. Piot, G. Casalis, F. Muller, and C. Bailly, "Investigation of the PSE approach for subsonic and supersonic hot jets. Detailed comparisons with LES and linearized Euler equations results," Int. J. Aeroacoust. 5, 361-393 (2006).

${ }^{13}$ K. Gudmundsson and T. Colonius, "Instability wave models for the near-field fluctuations of turbulent jets," J. Fluid Mech. 689, 97-128 (2011).

${ }^{14}$ J. W. Nichols and S. K. Lele, "Global modes and transient response of a cold supersonic jet,” J. Fluid Mech. 669, 225-241 (2011).

${ }^{15}$ K. Mohseni, T. Colonius, and J. Freund, "An evaluation of linear instability waves as sources of sound in a supersonic turbulent jet," Phys. Fluids 14, 3593-3600 (2002).

16 J. Freund, "Noise sources in a low Reynolds number jet at Mach 0.9," J. Fluid Mech. 438, 277-305 (2001).

${ }^{17}$ V. Suponitsky, N. Sandham, and C. Morfey, "Linear and nonlinear mechanisms of sound radiation by instability waves in subsonic jets," J. Fluid Mech. 658, 509-538 (2010).

${ }^{18}$ C. K. W. Tam and T. Chen, "Turbulent mixing noise from supersonic jets," AIAA J. 32, 1774-1780 (1994).

${ }^{19} \mathrm{C}$. Tam and F. Hu, "On the three families of instability waves of high-speed jets," J. Fluid Mech. 201, 447-483 (1989).

${ }^{20}$ H. Oertel, "Mach wave radiation for hot supersonic jets investigated by means of the shock tube and new optical techniques," in Proceedings of the 12th International Symposium on Shock Tubes and Waves (Magnes Press., Jerusalem, 1980), edited by A. Lifshitz and J. Rom, pp. 266-275.

${ }^{21}$ H. Salwen and C. Grosch, "The continuous spectrum of the Orr-Sommerfeld equation. Part II. Eigenfunction expansions," J. Fluid Mech. 104, 445-465 (1981).

${ }^{22}$ D. Hill, "Adjoint systems and their role in the receptivity problem for boundary layers," J. Fluid Mech. 292, 183-204 (1995).

${ }^{23}$ A. Tumin, "Three-dimensional spatial normal modes in compressible boundary layers," J. Fluid Mech. 586, 295-322 (2007).

${ }^{24}$ E. Mollo-Christensen, "Jet noise and shear flow instability seen from an experimenter's viewpoint," J. Appl. Mech. 34, 1-7 (1967)

25 J. T. C. Liu, "Developing large-scale wavelike eddies and the near jet noise field,” J. Fluid Mech. 62, 437-464 (1974).

${ }^{26}$ P. Morris, M. Giridharan, and G. Lilley, "On the turbulent mixing of compressible free shear layers," Proc. R. Soc. London, Ser. A 431, 219-243 (1990)

${ }^{27}$ T. Suzuki and T. Colonius, "Instability waves in a subsonic round jet detected using a near-field phased microphone array," J. Fluid Mech. 565, 197-226 (2006).

${ }^{28}$ R. H. Schlinker, J. C. Simonich, R. A. Reba, T. Colonius, and F. Ladeinde, "Supersonic jet noise from round and chevron nozzles: Experimental studies," AIAA Paper 2009-3257, 2009.

${ }^{29}$ G. A. Brès, J. W. Nichols, S. K. Lele, and F. E. Ham, "Towards best practices for jet noise predictions with unstructured large eddy simulations," AIAA Paper 2012-2965, 2012.

${ }^{30}$ T. Herbert, "Parabolized stability equations," Annu. Rev. Fuid Mech. 29, 245-283 (1997).

${ }^{31}$ K. Gudmundsson, "Instability wave models of turbulent jets from round and serrated nozzles," Ph.D. thesis, California Institute of Technology, 2010.

${ }^{32}$ F. Li and M. R. Malik, "Spectral analysis of parabolized stability equations," Comput. Fluids 26, 279-297 (1997). 
${ }^{33}$ C. Grosch and H. Salwen, "The continuous spectrum of the Orr-Sommerfeld equation. Part I. The spectrum and the eigenfunctions," J. Fluid Mech. 87, 33-54 (1978).

${ }^{34}$ A. M. Tumin and A. V. Fedorov, "Spatial growth of disturbances in a compressible boundary layer," J. Appl. Mech. Tech. Phys. 24, 548-554 (1983).

${ }^{35}$ A. M. Tumin and A. V. Fedorov, "Excitation of instability waves in a boundary layer on a vibrating surface," J. Appl. Mech. Tech. Phys. 24, 670-674 (1983).

${ }^{36}$ A. Fedorov and A. Tumin, "Initial-value problem for hypersonic boundary-layer flows," AIAA J. 41, 379-389 (2003).

${ }^{37}$ A. E. Gill, "Instabilities of top-hat jets and wakes in compressible fluids," Phys. Fluids 8, 1428-1430 (1965).

${ }^{38}$ C. Tam and D. Burton, "Sound generated by instability waves of supersonic flows. Part 2. Axisymmetric jets," J. Fluid Mech. 138, 273-295 (1984).

${ }^{39}$ F. Bertolotti and T. Colonius, "On the noise generated by convected structures in a Mach 0.9 hot, turbulent jet," AIAA Paper 2003-1062, 2003.

${ }^{40}$ P. Balakumar and M. R. Malik, "Discrete modes and continuous spectra in supersonic boundary layer," J. Fluid Mech. 239, 631-656 (1992).

${ }^{41}$ R. Briggs, Electron-Stream Interaction with Plasmas (MIT Press, Cambridge, 1964).

${ }^{42}$ D. G. Crighton and P. Huerre, "Shear-layer pressure fluctuations and superdirective acoustic sources," J. Fluid Mech. 220, 355-368 (1990).

${ }^{43}$ D. Rodríguez, A. Sinha, G. A. Brès, and T. Colonius, "Parabolized Stability Equation models in turbulent supersonic jets," AIAA Paper 2012-2117, 2012.

${ }^{44}$ D. Rodríguez, A. Samanta, A. Cavalieri, T. Colonius, and P. Jordan, "Parabolized Stability Equation models for predicting large-scale mixing noise of turbulent round jets," AIAA Paper 2011-2838, 2011.

${ }^{45}$ D. Juvé, M. Sunyach, and G. Compte-Bellot, "Intermittency in the noise emission in subsonic cold jets," J. Sound Vib. 71, 319-332 (1980).

${ }^{46}$ J. I. Hileman, B. S. Thurow, E. J. Caraballo, and M. Samimy, "Large-scale structure evolution and sound emission in high-speeds jets: real-time visualization with simultaneous acoustic measurements," J. Fluid Mech. 544, 277-307 (2005).

${ }^{47}$ K. Thompson, "Time dependent boundary conditions for hyperbolic systems," J. Comput. Phys. 68, 1-24 (1987).

${ }^{48}$ K. Mohseni and T. Colonius, "Numerical treatment of polar coordinate singularities," J. Comput. Phys. 157, 787-795 (2000).

${ }^{49} \mathrm{~W}$. Arnoldi, "The principle of minimized iterations in the solution of the matrix eigenvalue problem," Q. Appl. Math. 9, 17-29 (1951).

${ }^{50}$ Y. Saad, Numerical Methods for Large Eigenvalue Problems (Manchester University Press, 1992).

${ }^{51}$ P. R. Amestoy, I. S. Duff, J.-Y. L'Excellent, and J. Koster, “A fully asynchronous multifrontal solver using distributed dynamic scheduling," SIAM J. Matrix Anal. Appl. 23, 15-41 (2001). 